\title{
Globular Clusters: Low Mass Stars, Still No Brown Dwarfs!
}

\author{
Guido De Marchi \\ European Space Agency, Space Telescope Operations Division, 3700 San \\ Martin Drive, Baltimore MD 21218, USA
}

\begin{abstract}
In spite of all the attempts to find them, no one has yet detected any brown dwarf in a globular cluster. Although powerful instruments such as the VLT and Advanced Camera could further push the frontiers of this search, globular clusters will probably hold tight to their secrets for a while longer. Nonetheless, the search for very low mass stars in globular clusters has taught us a lot about their original mass distribution (IMF) and its evolution in time. I shall review the results of an investigation carried out over what is presently the largest, most homogeneous sample, and discuss the reasons suggesting that: 1. dynamical evolution (internal and external) has reshaped the cluster mass function over time, but the imprint of the IMF is still visible; 2 . the IMF appears to vary very little from cluster to cluster; 3 . the most likely functional form of the IMF is that of a power law that rises to a peak at $\sim 0.3 \mathrm{M}_{\odot}$ and tapers off at smaller masses.
\end{abstract}

\section{Introduction}

A few months ago I happened to mention to my friend and colleague João Alves (known to most of the delegates in the audience) that I should have come to this symposium to illustrate the status of the search for brown dwarfs (BD) in globular clusters (GC). He laughingly remarked that, since so little is known on this topic and no observational data exist, I should just present an empty viewgraph and quickly move to the beach! It is true that the first $\mathrm{BD}$ in a GC has yet to be discovered and, even when we shall finally detect some, we expect them to account for a marginal fraction of the total mass of these clusters (see e.g. Chabrier \& Méra 1997). Ten years ago, however, when we started this investigation with the Hubble Space Telescope (HST), expectations were radically different. Deep ground based observations were suggesting that the mass function (MF) of GC stars should be rising steeply below $\sim 1 \mathrm{M}_{\odot}$, with a power-law index as high as $\alpha=3$ or more, all the way through to the H-burning limit (Richer et al. 1991). And because a forming star does not know exactly which its final mass will be on the main sequence (MS), it seemed natural to assume that this steep trend would continue well into the sub-stellar domain. The refurbished HST has proved this hypothesis wrong, revealing no BD so far, but the bounty of data collected in this way has allowed us to develop a much clearer picture of the stellar initial mass function (IMF). 
The IMF is a critical ingredient in our understanding of a large number of basic astronomical phenomena such as the formation of the first stars, galaxy formation and evolution and the determination of the absolute star formation rate. It also plays a dominant role in any star formation theory as the end result of molecular cloud contraction and fragmentation. The slope of the MF at the lowest mass end of the stellar MS and, in particular, whether or not there is a turn-over at the lowest masses before the H-burning limit and whether or not the IMF is universal or rather depends on the initial physical conditions in the natal environment are critical open issues at present.

In principle, the measurement of the stellar MF in this low mass range would be optimally carried out on the youngest open clusters now being formed like the Orion Nebula or IC348, for example. A number of problems limit the accuracy of this technique, however. These include the small number of cluster members spread out over large areas of sky, contamination from foreground objects, completeness limits that are currently uncomfortably close to the end of the main sequence, the presence of unresolved binaries, uncertain corrections for extinction and, especially, the mass-age degeneracy of the conversion from luminosity to mass for such young stellar populations.

Globular clusters, on the other hand, are free of most of these problems and, thus, represent another important way to deduce the stellar IMF and properly answer some of the above questions. They offer a large statistically significant sample of relatively bright, coeval, equidistant stars with, in most cases, relatively small variations of chemical composition and extinction within each cluster. They were all formed very early in the history of the Galaxy and there is no evidence of subsequent star formation episodes (but see Ferraro, Bellazzini $\&$ Pancino 2002 as regards $\omega$ Cen). The binary fraction outside the core is less than $10 \%$ (Albrow et al. 2001) and has an insignificant effect on the measured luminosity function (LF). Mass segregation is a relatively straightforward and well understood phenomenon quantifiable by simple Michie-King models. The only potentially serious obstacle is related to the possible modification of the IMF by the effect of tidal interactions with the Galaxy potential. This interaction, integrated over the orbit and time, is expected to slowly decrease the slope of the global mass function of the cluster (Vesperini 1998) thereby effectively masking the original IMF from our present day observations, no matter how precise and detailed they are.

Since deep LF of a dozen GC in our Galaxy's halo have now been accurately measured, we are in a good position to address observationally the issue of if and, possibly, how the interaction history of these clusters, whose Galactic orbits are reasonably well known, affects their LF in the mass range where the signature is expected to be most significant. In this paper, we show that LF obtained at or just beyond the half-light radius of these clusters surveyed are completely insensitive to this history and that they can indeed be used to deduce an uncontaminated stellar IMF below $1 \mathrm{M}_{\odot}$ for these stars.

\section{Observational data}

The main characteristics of the data used for this study have been summarised elsewhere (Paresce \& De Marchi 2000). The LF of the 12 clusters in this sample 


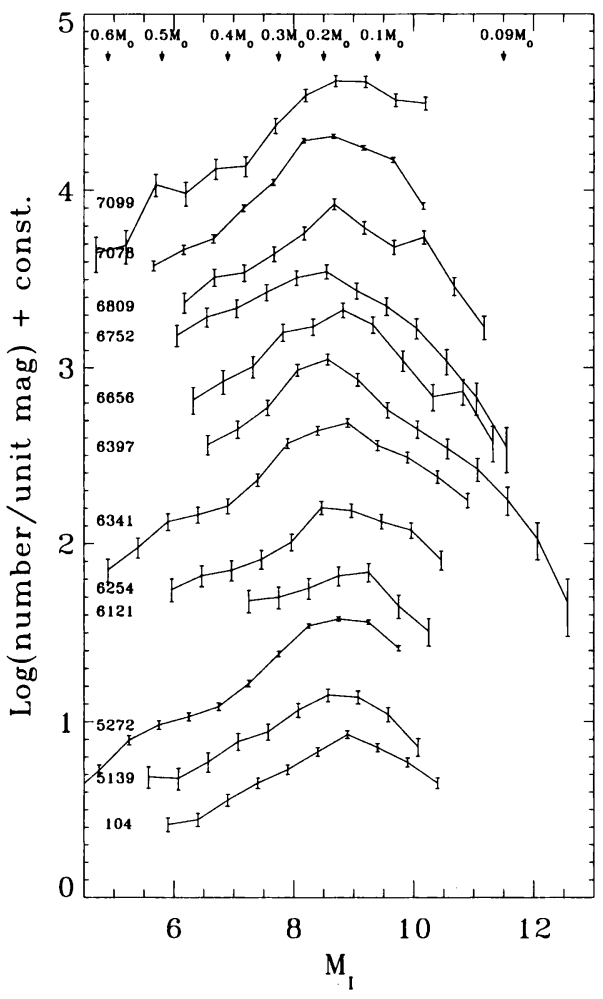

Figure 1. Luminosity functions of the 12 globular clusters in our sample. See Paresce \& De Marchi (2000) for more details on the data.

are shown in Figure 1, whilst Figure 2 shows the log-normal (LN) and tapered power law (TPL) distributions that accurately reproduce the measured LF over the whole magnitude range spanned by the observations (the fit is shown in Figure 3). The conversion of the MF to LF is performed using the appropriate mass-luminosity (ML) relation computed by Baraffe et al. (1997), whose validity has been recently confirmed independently by Cassisi, Castellani \& Ciarcelluti (2001). Solid lines in Figure 2 mark the portion of the MF that has been fitted to the data, whilst the dashed lines represent the extrapolation of the same MF to fill in the range $0.09-0.75 \mathrm{M}_{\odot}$. The LN MF is characterised by only two parameters namely the characteristic mass $m_{\mathrm{c}}$ and the standard deviation $\sigma$ and takes on the form $\ln f(\log m)=A-\frac{\left[\log \left(m / m_{c}\right)\right]^{2}}{2 \sigma^{2}}$, where $A$ is a normalisation constant. The TPL MF is characterised by an index $\alpha$ that we have assumed to be $\alpha=2.35$ (the canonical Salpeter's IMF), by a peak mass $m_{p}$, and by a tapering exponent $\beta$. The analytical form of the TPL MF is $f(m)=B \times m^{-\alpha} \times$ $\left[1-e^{\left(m / m_{p}\right)^{-\beta}}\right]$, where $B$ is again a normalisation constant.

The average values of the parameters for this sample of clusters are for the $\mathrm{LN}$ case $\left\langle m_{c}\right\rangle=0.32 \pm 0.03$ and $\langle\sigma\rangle=0.34 \pm 0.04$ whilst for the TPL case, having assumed $\alpha=2.35$, they are $\left\langle m_{p}\right\rangle=0.34 \pm 0.03 \mathrm{M}_{\odot}$ and $\langle\beta\rangle=2.6 \pm 0.2$. The uncertainties accompanying $\left\langle m_{c}\right\rangle,\langle\sigma\rangle,\left\langle m_{p}\right\rangle$, 

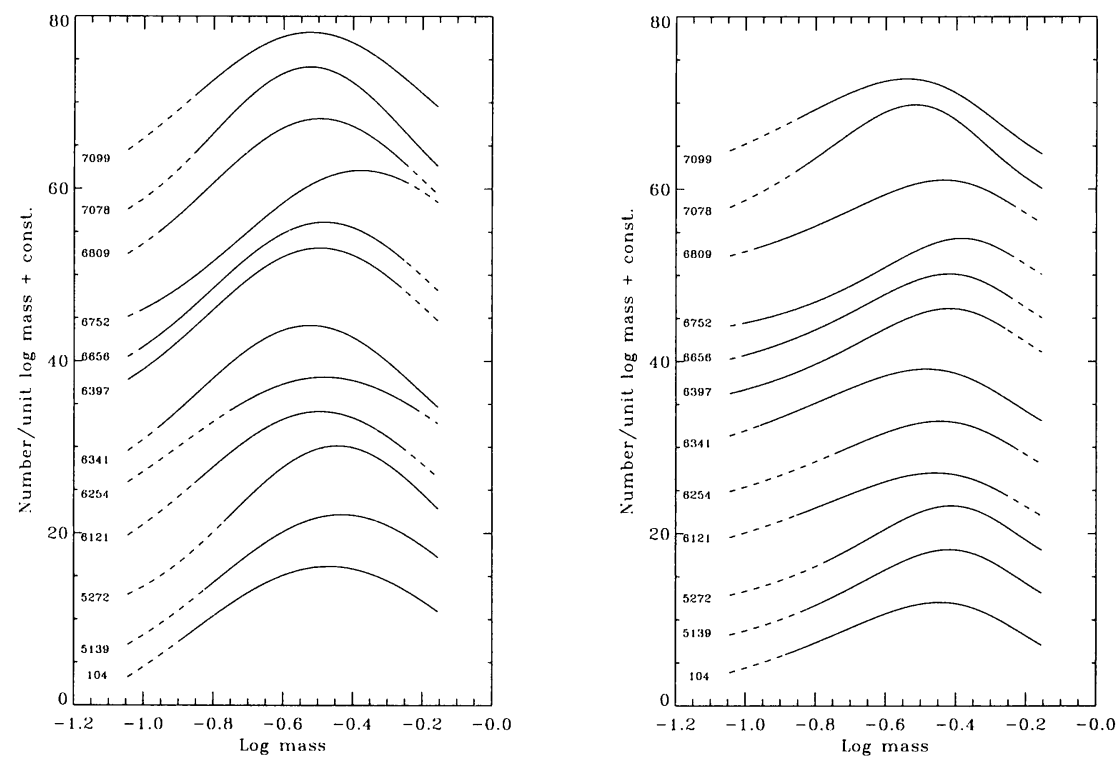

Figure 2. The log-normal (left-hand panel) and tapered power-law (right-hand panel) MF that best fit the HST-based LF in Figure 1. The scale is linear along the y axis.
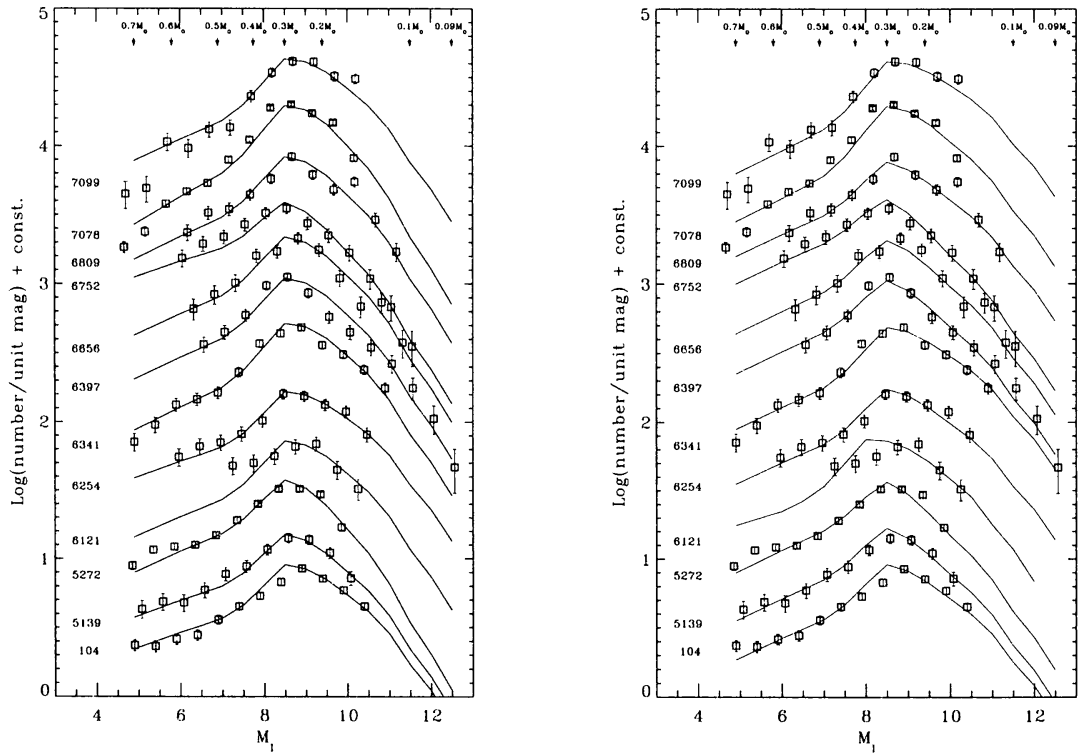

Figure 3. The data of Figure 1 are compared here with the theoretical LF corresponding to the MF of Figure 2 . 


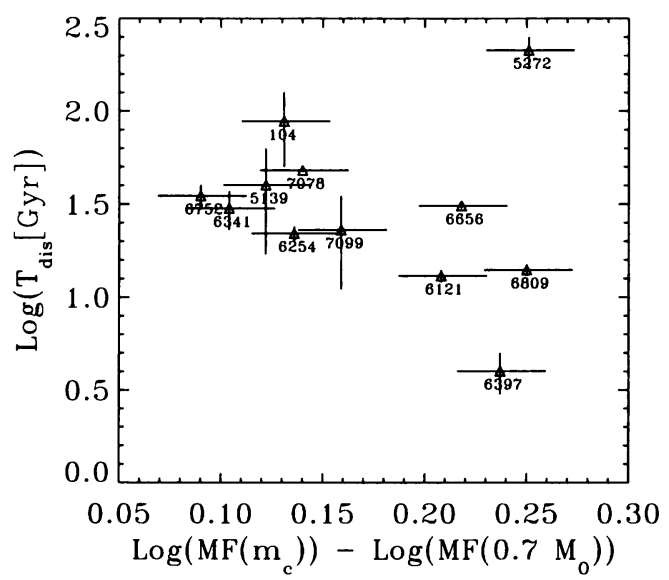

Figure 4. The time to disruption in Gyr as calculated by Gnedin \& Ostriker (1997) is shown here as a function of the index $\Delta \log N$ describing the shape of the MF in Figure 2 (left-hand panel). Error bars define the $\pm 3 \sigma$ uncertainty.

and $\langle\beta\rangle$ represent the scatter of the values of these parameters around their mean. As an example, the individual values of $m_{c}$ and $\sigma$ are given for each cluster in Table 1. It should be noted that the relatively small values of $\sigma$ imply that, beyond the peak, the MF drops not only in the logarithmic plane, but also in linear units, i.e. the number of stars per unit mass decreases with decreasing mass below the peak. The large value of $\beta$ leads to the same result in the TPL case. We note in passing that, although both the LN and the TPL MF, once converted into LF, agree well with the data over the whole range spanned by the HST observations, at higher masses the two functions depart significantly from each other, with the TPL becoming practically indistinguishable from a Salpeter power-law function of index $\alpha=2.35$.

\section{Discussion and final remarks}

A simple, unbiased measure of the steepness of the rise to the maximum of LN or TPL MF shown in Figures 2 that does not depend on any preconceived notion on the shape of the MF is $\Delta \log N$, defined as the logarithmic ratio of the number of lower to higher mass stars taken from the MF at the peak $\left(m_{c}\right.$ or $m_{p}$ ) and at $0.7 \mathrm{M}_{\odot}$. This is probably the most convenient parameter to describe the region of the mass distribution most likely to be affected by external and internal dynamics and is listed in Table 1 for each cluster. Another advantage of $\Delta \log N$ is that it is defined in a mass range where the stellar surface structure is best understood and all presently available models for the ML relation are in good agreement with each other (Silvestri et al. 1998) and is, in consequence, least likely to be subject to uncertainties due to the LF to MF conversion.

Table 1 lists the main physical parameters of the clusters surveyed so far. Since the main objective here is to search for the signature of a cluster's dynam- 
ical history on its low mass MF, this table includes whatever is known about its orbit in the Galaxy. The space motion data were obtained from the work of Dauphole et al. (1996) and Odenkirchen et al. (1997). These data can be used in a theoretical model to determine the change with time of the cluster's main characteristics such as total mass, MF and LF, tidal radius, central concentration, relaxation time, etc. Both $\mathrm{N}$-body and Fokker-Planck models of increasing sophistication have been used recently to compute such evolution (Takahashi \& Portegies Zwart 2000; Gnedin, Hernquist \& Ostriker 1999; Vesperini 1998, 1997; Vesperini \& Heggie 1997; Gnedin \& Ostriker 1997; Capriotti \& Hawley 1996; Murali \& Weinberg 1997). Although different authors use different initial conditions and approximations to the complex tidal interaction mechanisms, the generally physically plausible final result is a flattening of an assumed powerlaw low mass MF with time due to the preferential evaporation of lower mass stars forced by two-body relaxation out to the cluster periphery, where the evaporation process is accelerated by tidal shocks.

A direct calculation of this phenomenon for a specific cluster orbit has not been carried out yet but an indirect indication at least of the magnitude of the effect can be gleaned from the recent calculations of the time to disruption $T_{d}$ of specific clusters carried out by Gnedin \& Ostriker (1997) and by Dinescu, Girard \& Van Altena (1999). These times are given in Gyr in Table 1 (assuming a value of $10 \mathrm{Gyr}$ for a Hubble time) where the two values of the total destruction rate given by Gnedin \& Ostriker (1997) for the two Galactic models used in their calculations have been averaged in column (10). The observed clusters cover quite a large range of $T_{d}$, whose values should in principle be regarded as upper limits to the true $T_{d}$, as both authors treat the internal dynamical evolution of the clusters by using single-mass Michie-King models and, thus, tend to underestimate the effects of mass segregation. An inspection of Table 1 shows that no one particular orbital parameter or the cluster mass by itself is sufficient to foretell what the fate of the cluster will be. Even, for example, the cluster's perigalactic distance or its height above the plane are not well correlated with $T_{d}$. This means that the overall impact of the repeated bulge and disc shocks on the cluster over its lifetime is not easily predictable from a simple glance at the orbital parameters but only from the use of calculations over the entire orbit such as those mentioned above.

One would thus expect that the clusters with the largest times to destruction $T_{d}$, or those that have suffered the least tidal disruption, should have the largest low to high mass number ratio $\Delta \log N$. The actual situation is shown in Figure 4 where the time to disruptions is plotted as a function of $\Delta \log N$. The best linear fit to this distribution is a straight line intercepting the abscissae at $\Delta \log N=0.07 \pm 0.03$ and having a slope of $0.01 \pm 0.02$. A vertical line drawn at $\Delta \log N \simeq 0.09$, however, would still give an acceptable fit. Within the errors, then, there is no discernible trend in this direction and the conclusion at this point is, therefore, quite clear: the global MF of the clusters in this sample shows no evidence of evolution with time within the quoted errors.

In light of the very small range spanned by $m_{c}$ and $\sigma$ or by $m_{p}$ and $\beta$ as deduced for the 12 clusters, the conclusion is that a single form of the MF can easily reproduce all the 12 deep LF obtained so far. Furthermore, since there is no obvious dependence on dynamical history over an extremely wide range of 
conditions, this MF is most likely to represent the initial distribution of stellar masses in the cluster, namely the IMF. When observations are refined in the future and a larger sample is built, it might become possible to assess whether the small cluster-to-cluster differences that are still seen in the MF are due to the interaction with the tidal field of the Galaxy or whether they reflect different conditions at birth. And indeed, the very fact that, at least for GC, the IMF has a scale mass opens up the exciting new possibility of using the IMF to probe the physical conditions of the molecular clouds out of which stars form, since it is conceivable that pressure, temperature and density will cast their signature on the IMF, thus eventually determining its peak mass and width.

To properly explore this region of the parameter space, one needs a larger and better data sample, covering not only GC, but most importantly younger clusters, where one has access to higher masses and metallicity. This investigation should yield a bountiful harvest of information on the stellar IMF in the near future. Of particular importance in this endeavour, will be securing a sufficiently large, clean sample of stars of the same physical and kinematical type in a wide variety of environments and ages and to develop the most accurate models of their energy output as a function of mass.

\section{References}

Albrow, M., et al. 2001, ApJ, 559, 1060

Baraffe, I., Chabrier, J., Allard, F., Hauschildt, P. 1997, A\&A, 327, 1054

Capriotti, E., Hawley, S. 1996, ApJ, 464, 765

Cassisi, S., Castellani, V., Ciarcelluti, P. 2001, MmSAI, 72, 743

Chabrier, G., Méra, D. 1997, A\&A, 328, 83

Dauphole, B., et al. 1996, A\&A, 313, 119

Dinescu, D., Girard, T., Van Altena, W. 1999, AJ, 117, 1792

Djorgovski, S. 1993, in Structure and Dynamics of Globular Clusters, ASP Conf.

Ser. 50, ed. S. Djorgovski and G. Meylan (San Francisco: ASP), 373

Ferraro, F., Bellazzini, M., Pancino, E. 2002, ApJ, 573, L95

Gnedin, O., Hernquist, L., Ostriker, J. 1999, ApJ, 514, 109

Gnedin, O., Ostriker, J. 1997, ApJ, 474, 233

Murali, C., Weinberg, M. 1997, MNRAS, 291, 717

Odenkirchen, M., Brosche, P., Geffert, Tucholke, H. 1997, NewA, 2, 477

Paresce, F., De Marchi, G. 2000, ApJ, 534, 870

Richer, H., et al. 1991, ApJ, 381, 147

Silvestri, F., Ventura, P., D'Antona, F., Mazzitelli, I. 1998, ApJ, 509, 192

Takahashi, K., Portegies Zwart, S. 2000, ApJ, 535, 759

Vesperini, E. 1997, MNRAS, 287, 915

Vesperini, E. 1998, MNRAS, 299, 1019

Vesperini, E., Heggie, D. 1997, MNRAS, 289, 898

Webbink, R. 1985, IAU Symp, 113, 541 


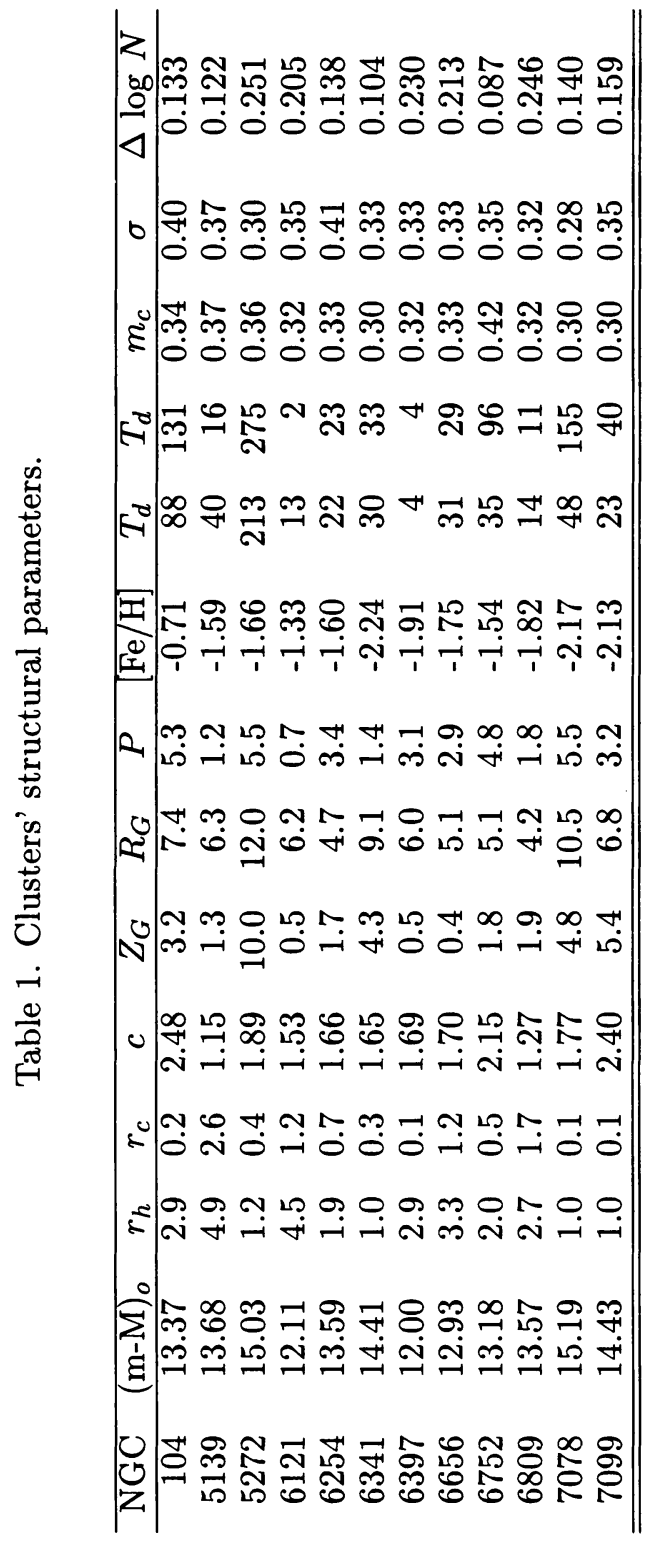

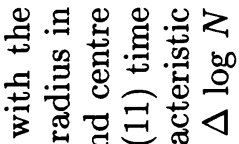

○ స

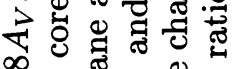
赵

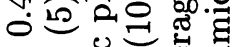
$+\tau$ $>\approx$ ส

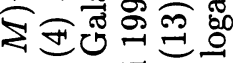

ह ง

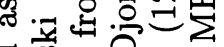
马ु :

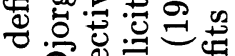
르용 ฮี.

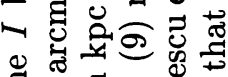

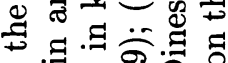

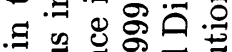

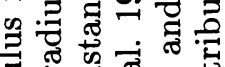

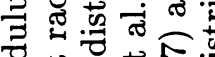

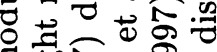
घ도워 Ч สี ฮี่ 苟 ฮูอ

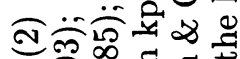
牙

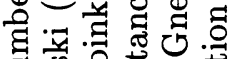

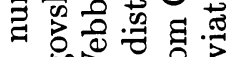
0 幽.

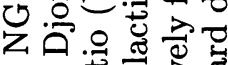
三 $\ddot{\text { is }}$

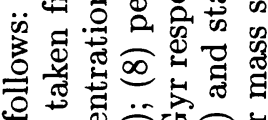
\& 仓 శ ซี่

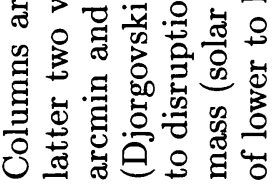

